Int. J. Dev. Biol. 60: 181-188 (2016)

doi: $10.1387 / \mathrm{ijdb} .150317 \mathrm{nr}$

\title{
Nucleoporin gene expression in Xenopus tropicalis embryonic development
}

\author{
NOOREEN REZA, MUSTAFA K. KHOKHA and FLORENCIA DEL VISO* \\ Program in Vertebrate Developmental Biology. Department of Pediatrics and Genetics, \\ Yale University School of Medicine, New Haven, CT, USA
}

\begin{abstract}
Nucleoporins (nups) compose the structure of the nuclear pore complex (NPC) of all cells, but several studies have illuminated nucleoporins' additional roles in development and the cell cycle. However, a comprehensive study of nup expression in embryonic development has not yet been reported. We synthesized antisense probes for all nup genes and used whole-mount in situ hybridization techniques to determine the expression pattern of all members of the nup family of genes at three different developmental stages in Xenopus tropicalis. We found that the expression of nups was not ubiquitous in embryos, but was localized to specific and distinguishable anatomical structures at all three stages tested. We also found that the expression patterns for nups within the same subcomplexes were not necessarily identical. Thus, nup expression is subject to a significant level of regulation during development. These results provide new information for functional studies of nups to unravel their roles in embryonic development.
\end{abstract}

KEY WORDS: nups, $N P C$, nuclear pore complex, in situ hybridization

The nuclear membrane forms an essential boundary for the nucleus and is a major site of macromolecular traffic in the cell, necessitating a mechanism for selective transport (Lusk et al., 2007). The nuclear pore complex (NPC) is a cylindrical array of proteins, embedded in the nuclear envelope, which creates a portal for macromolecular transport. The structure of the NPC has been analyzed and categorized into a cytoplasmic ring, a central spoke ring, and a nuclear ring (Grossman et al., 2012). Each of these subunits is composed of proteins called nucleoporins (nups), which form different subcomplexes: transmembrane, scaffold (outer and inner ring subcomplexes), cytoplasmic (ring/ linker), nuclear basket, and channel nups. Transmembrane nups anchor the NPC to the nuclear envelope, while scaffold and central channel nups make up the central structure of the transport complex (Fig. 1A). Several cytoplasmic, channel and nuclear basket nups have phenylalanine-glycine (FG) repeats that assist in trafficking molecules. For instance, central FG-repeat nups arrayed in the central pore create the permeability barrier of the NPC (Onischenko and Weis, 2011). A new study has recently elucidated the underlying protein-protein interaction network within the NPC. Analyzing the X-rays crystallographics of scaffold nucleoporins enabled the construction of full-length atomic structures, ultimately leading to a massive composite structure of the NPC (Lin et al., 2016).
Aside from their role at the NPC, many nucleoporins have been shown to have functional roles in several cell processes influencing development (Raices and D'Angelo, 2012). For example, the Nup107-160 subcomplex is known to promote microtubule spindle assembly in mitosis by recruiting the gamma-tubulin ring complex to kinetochores (Mishra etal., 2010). Other studies have associated nups like Nup98 and Nup153 with functions as diverse as HIV-1 import or integration (Di Nunzio etal., 2013). In addition, nup188was identified as a candidate gene for Congenital Heart Disease, and its knockdown showed disrupted left-right patterning during Xenopus embryonic development (Fakhro et al., 2011). While multiple nucleoporins have been the sole subjects of functional studies, a broad analysis of nucleoporin expression has not been undertaken. In addition, given that nup proteins are necessary in all nuclei, a priori, we anticipated that nups would be ubiquitously expressed during embryonic development where frequent cell divisions are regularly increasing overall nuclear envelope length. However, we were surprised to see that nup188had localized expression (Fakhro et al., 2011). Moreover, we used ultra-high-frequency sampling of Xenopus embryos and absolute normalization of sequence reads

Abbreviations used in this paper: NPC, nuclear pore complex, nup, nucleoporin.

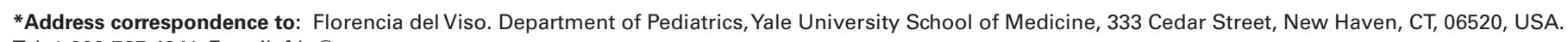
Tel: 1-203-737-4241. E-mail: fdv@stowers.org

Supplementary Material (one figure) for this paper is available at: http://dx.doi.org/10.1387/ijdb.150317nr

Accepted: 1 June 2016.

ISSN: Online 1696-3547, Print 0214-6282

(C) 2016 UPV/EHU Press

Printed in Spain 
to show gene expression profiles in absolute transcript numbers (Owens et al., 2016). Analyzing the nup expression profiles from this study we found dynamic temporal expression profiles during early development. Given these results, we sought to investigate the spatiotemporal expression of nups at different stages during embryonic development.

Using whole mount in situ hybridization, we analyzed the expression of all nups throughout Xenopus tropicalis development. In this study we show that nups are expressed in non-ubiquitous patterns across stages, revealing spatial regulation of nup expression during embryonic development.

\section{Results}

We began by analyzing an ultra-high resolution temporal expression profile. Using RNAseq and absolute normalization of mRNA levels, we calculated the number of transcripts per embryo during the first 66 hours post fertilization (stages 0 to 45) in $X$. tropicalis (Owens et al., 2016). We generated temporal expression profiles for each of the nup subcomplexes revealing dynamic expression (Fig. 2). Based on this data, we sought to investigate the spatial expression profiles in order to identify any spatiotemporal regulation.

To study nup spatial expression during embryonic development, we focused on three stage-ranges: stages 16-18, 26-28, and 33-36. These are benchmark times of embryonic development after initial germ layer specification and encompass much organogenesis. We examined expression in multiple important anatomical structures such as the neural tube, eye, heart and visceral (branchial) arches, as well as other structures (Fig. 1 B,C,D). We present our results organized by NPC subcomplex.

\section{Scaffold nucleoporins}

We detected strong signal of scaffold nups in the following regions: neural tube, head, eyes, visceral arches, dorso-lateral plate mesoderm, and ventral mesoderm. Variations in these expression patterns are discussed in further detail below.

\section{Outer ring: nups $37,43,85,96,107,133,160$, sec13, seh1}

At stages 16-18 all outer ring nups displayed expression in the anterior regions of the neural tube, and also near the eye anlages (Figs.1B and 3).

At stages 26-28, all nups in the subcomplex had signal in the eyes, midbrain and hindbrain. The eye signal was strong in nup37, 107, sec13and seh1. In addition, nups 37, 107 and sec13displayed strong signal in the forebrain. We also detected signal in the otic vesicle for all nups but nup43 and nup85. Nup133, sec 13 and seh1 also had signal in the tailbud. All nups in this subcomplex were detected in the dorso-lateral plate mesoderm region, where pronephros will develop, with the exception of nup37 and nup85 (Fig. 3, Im). At these stages, only seh1 was detected in the ventral mesoderm (Fig. 3, vm). Sec13 was also detected strongly in the cement gland (Fig. 3, c).

By stages 33-36, we detected strong signal for all nups in the head, eyes and visceral arches, with some variations in specific expression pattern. Nup133 had a unique expression pattern in the brain at these stages. There was strong signal detected in the mid to hindbrain boundary (the isthmus, Fig. 3 , i) whereas the immediate midbrain region showed comparatively weak signal; signal

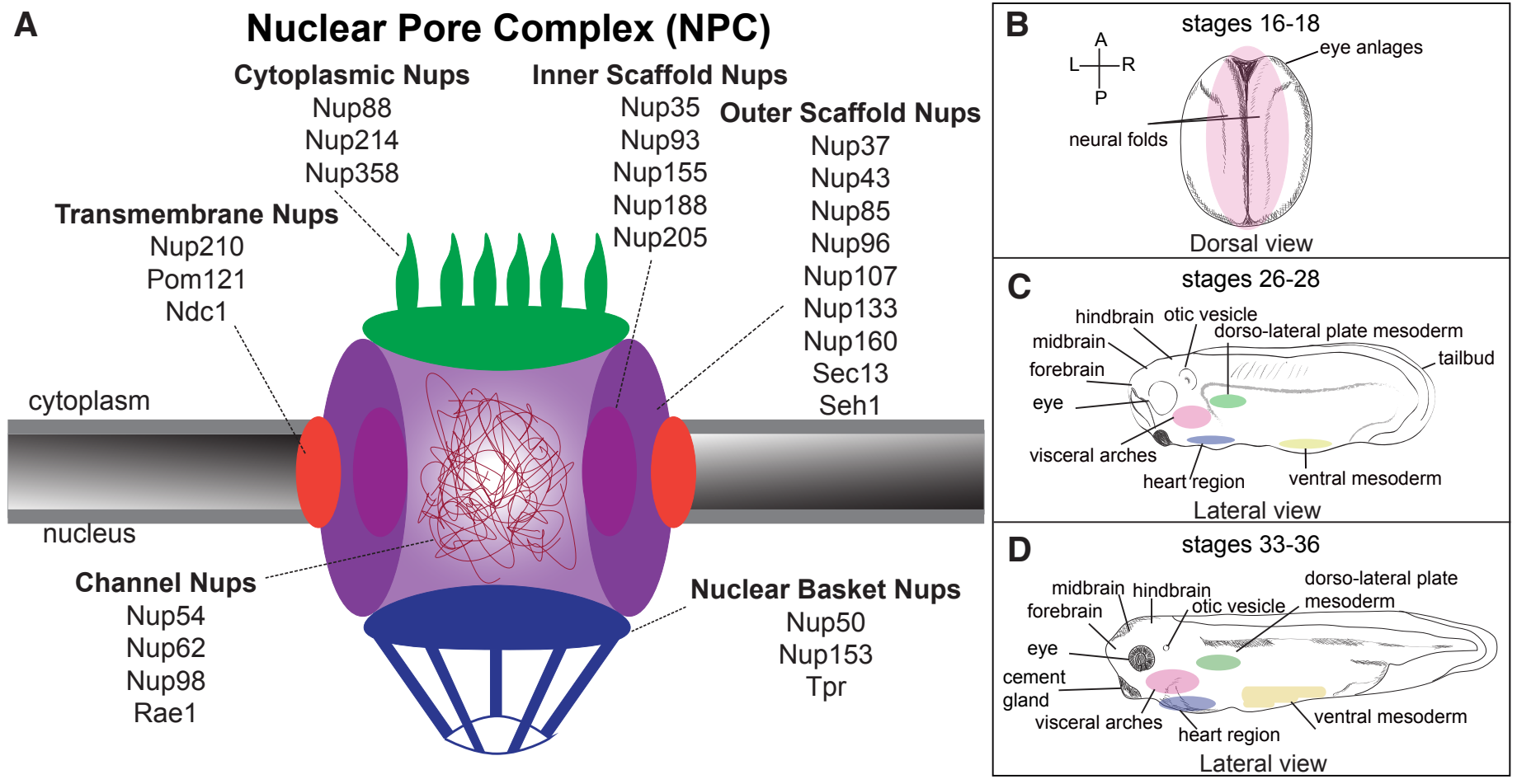

Fig. 1. (A) Schematic of Nuclear Pore Complex (NPC). The different subcomplexes that form the NPC are shown. (B,C,D) Schematic of X. tropicalis embryos at stages 16-18 (B), 26-28 (C), and 33-36 (D). The embryo schematics have relevant anatomical structures highlighted and labeled. A, anterior; $P$, posterior; $L$, left; $R$, right. 
again increased towards the forebrain. Otherwise, all members of the subcomplex displayed signal in the brain with the exception of nup43. All nups were detected in the dorso-lateral plate mesoderm, with the exception of nup37 and nup85. Signal in the heart progenitor field was detected for sec13 and seh1. Seh1 and sec13 also showed signal in the ventral mesoderm.

\section{Inner ring: nups 35, 93, 155, 188, 205}

At stages 16-18, similarly to the outer ring nups, all the inner ring genes displayed significant expression in the anterior and posterior region of the neural tube (Fig. 4).

At stages 26-28, all nups displayed signal in the eyes that was particularly strong in nups 93, 188 and 205. These three nups also share broad expression in the brain, visceral arches and otic vesicle. It is notable that nups 93, 188 and 205 display a common expression pattern at these stages, since these three nups were shown to interact with each other (Theerthagiri et al., 2010). Dorso-lateral plate mesoderm signal was detected for all the nups in this subcomplex, with the exception of nup35. Heart progenitor field signal was observed in nups 93 and 155. Ventral mesoderm signal was detected for nup155 only. All nups showed signal at the tailbud.

At stages 33-36, the presence of signal in the head, brain, eyes, and visceral arches was maintained. Nups 93 and 155 in particular showed an expression pattern in the brain similar to nup133 at these stages, with an area of strong signal in the isthmus (Fig. 4, i). Nups 93 and 188 had strong signal in the eye. Dorso-lateral plate mesoderm signal was detected in all the nups besides nup35 and nup205. Heart progenitor field signal was maintained in nups 93 and 155 (Fig. 4, h). At this stage we detected ventral mesoderm signal only in nup205.

\section{Channel nucleoporins: nups 54, 62, 98, rae1}

In general, we detected signal in the head, eyes, somites, otic vesicle and visceral arches of the channel nups. At stages 16-18, all genes showed signal in the anterior regions around the eye anlages (Fig. 5). Rae1 signal was detected strongly along the whole neural tube.

At stages 26-28, we detected signal in the brain, eyes, otic vesicle and visceral arches for all nups. The signal detected for nup62 and rae 1 was broader around the head region and not clearly restricted to the visceral arches. They also showed strong signal along the entire length of the neural tube and in the somites. Nup54 and nup98 were expressed in the tailbud. It is interesting to note that the Nup96 and Nup98 proteins derive from a single protein precursor that is cleaved yielding two individual proteins. Regarding mRNAs, the long nup98-nup96 mRNA precursor and another nup98 mRNA, derived from an alternatively spliced pre-mRNA coexist (Fontoura et al., 1999). Our probes were designed to hybridize to nup96and nup98separately; however due to the nature of these particular genes it is not possible to discern their individual expression pattern by in situ hybridization. As expected, these genes showed similar expression patterns (Figs. 3 and 5).

At stages 33-36, the trend of general expression in the brain and eyes was sustained. Nup54 expression pattern in the brain is similar to

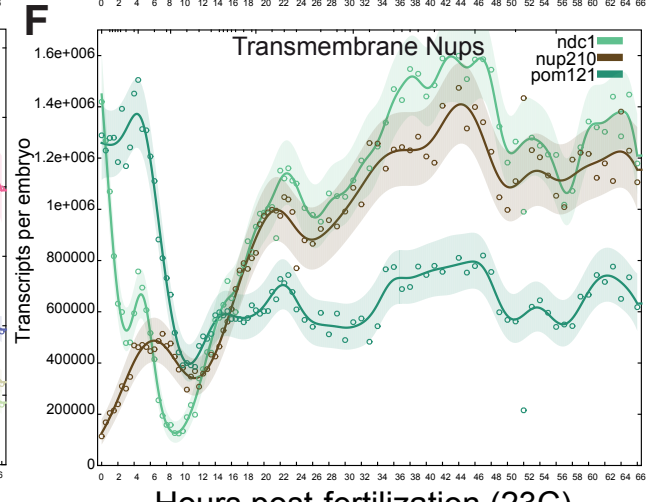

Hours post-fertilization (23C)
Fig. 2. RNA-seq profiles of nups during $X$. tropicalis development. Outer ring (A), inner ring (B), channe/(C), cytoplasmic (D), nuclear basket(E) and transmembrane nucleoporins (F). Graphs show number hours post fertilization (horizontal axis). Nieuwkoop and Faber (NF) developmental stage is shown on the top. Shaded regions mark Gaussian process $95 \% \mathrm{Cls}$ of the data. of transcripts per embryo from 0 to 66 
nup133 (Fig. 3) with strong signal in the isthmus (Fig. 5, i). All genes in this subcomplex showed signal in the visceral arches and otic vesicle. We detected signal in the tail for nup54. We also detected strong signal in the neural tube and somites for nup62 and rae1 as in previous stages.

\section{Cytoplasmic ring and nuclear basket nucleoporins: nups 50,} 88, 153, 214, 358, tpr

These subcomplexes presented signal in the brain, eyes, visceral arches, dorsolateral plate mesoderm, heart, and otic vesicle. Variations in these observations are discussed below (Fig. $6 \mathrm{~A}, \mathrm{~B}$ ).

At stages 16-18, most of the genes showed signal in the anterior

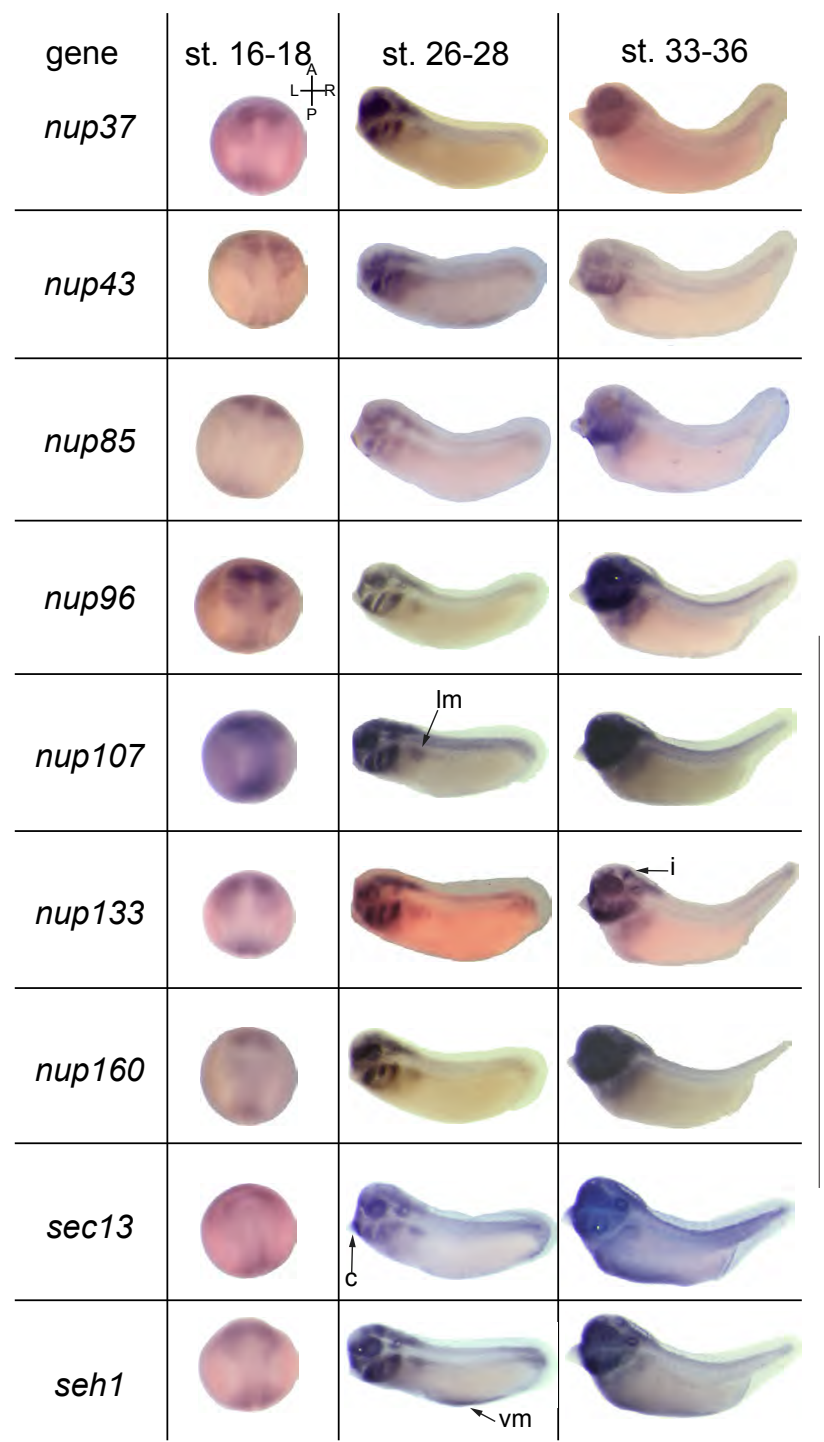

regions around the eye anlages coupled with visible expression in the posterior regions (Fig. 6B).

At stages 26-28, all nups were detected in the brain, eyes and visceral arches. We detected signal in the neural tube for nup50 and tpr. There was also signal detected in the otic vesicle for nup50. Some signal was observed near the tail for nup153. All the nups in these subcomplexes displayed dorso-lateral plate mesoderm signal with the exception of tpr. Nup88 was also strongly detected in the cement gland (Fig. 6A).

At stages 33-36 we detected brain expression for most genes. For nup88, in particular, we detected strong signal in the isthmus, similar to nup133 (Fig. 6A). There was signal detected in the otic vesicle for nups 50, 88 and 153 . We also observed dorso-lateral plate mesoderm expression in all genes of these subcomplexes. Heart progenitor field signal was observed for nup50, 214 and tpr (Fig. 6B). Nup153 and nup88 were the only genes detected in the ventral mesoderm in these subcomplexes, and only at these stages.

\section{Transmembrane nucleoporins: nup210, pom121, ndc1}

At stages 16-18, these three nups exhibited signal in the anterior regions surrounding the eye anlages and posterior region of the neural tube (Fig. 7).

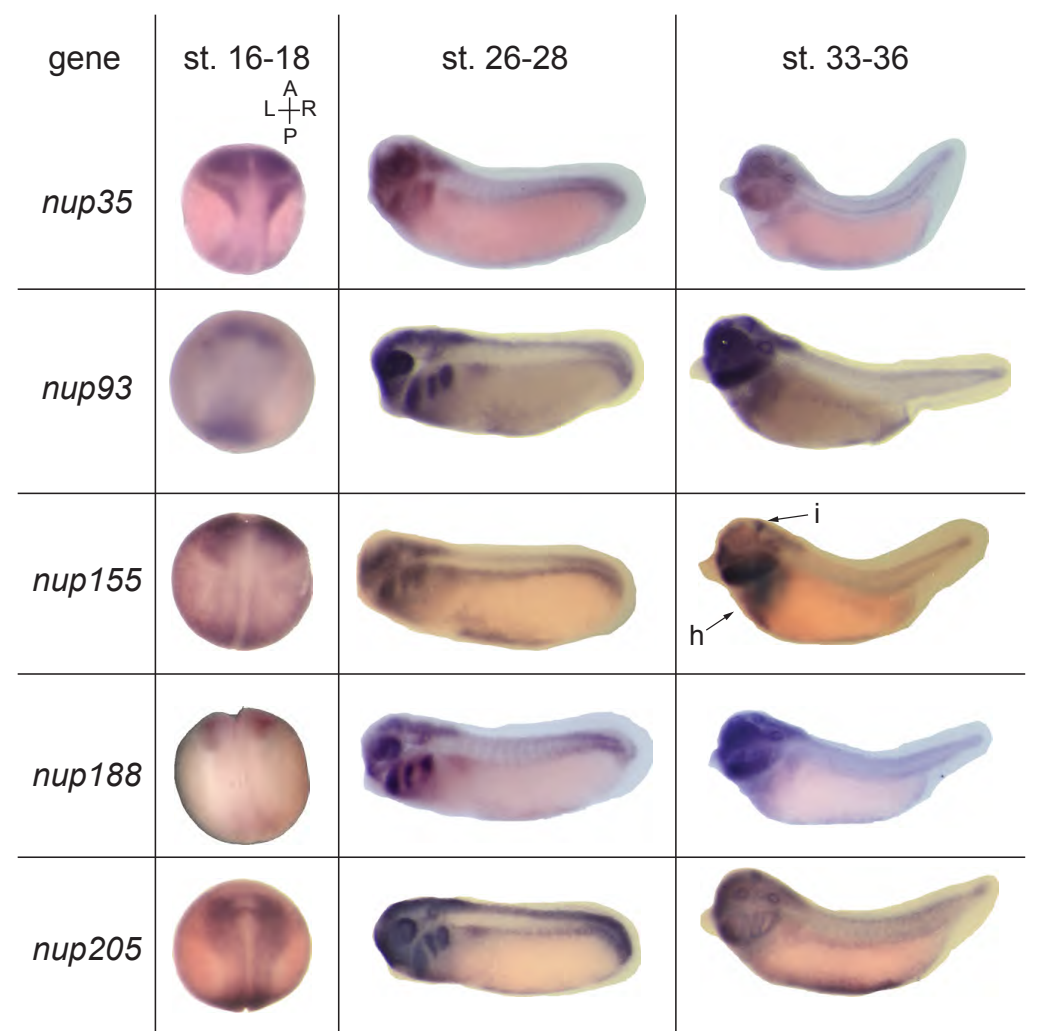

Fig. 3 (Left). Expression of outer ring scaffold nups in developing $\boldsymbol{X}$. tropicalis embryos. Expression was analyzed by whole mount in situ hybridization at 3 different stages of X. tropicalis development (16-18, 26-28 and 33-36, columns). Signal was detected for all the nups in the anterior regions of the neural tube and near the eye anlages at stages 16-18. At stages 26-28 and 33-36, signal was detected for all nups in the brain, eyes, and visceral arches. C, cement gland; i, isthmus or mid to hindbrain boundary; Im, dorsolateral plate mesoderm; vm, ventral mesoderm. A: anterior, P: posterior, L: left, R: right.

Fig. 4 (Right). Expression of inner ring scaffold nups in developing $\boldsymbol{X}$. tropicalis embryos. Expression was analyzed by whole mount in situ hybridization at 3 different stages of $\mathrm{X}$. tropicalis development (16-18, 26-28 and 33-36, columns). All nups were detected in the neural plate region at stages 16-18 and in the head, visceral arches and eyes at stages 26-28 and 33-36. h, heart progenitor field; i, isthmus or mid to hindbrain boundary. A: anterior, P: posterior, L: left, R: right. 
At stages 26-28 there was signal detected for all three genes in the brain, eyes and visceral arches (Fig. 7). For pom121 we detected signal in the somites. Nup210 and ndc1 showed tailbud signal. There was also signal at the otic vesicle for ndc 1 and nup210. In contrast to the other subcomplexes, we did not detect strong signal at the dorso-lateral plate mesoderm for these genes. However, strong ventral mesoderm signal was detected for nup210 and ndc1 at these stages.

At stages 33-36, we continued to observe general expression in the eyes, brain, otic vesicle and visceral arches for all three genes (Fig. 7). We observed ndc1 signal along the length of the neural tube. Ventral mesoderm expression was observed for nup210 and $n d c 1$.

\section{$R T-P C R$ validation}

To assess if the specific expression detected by in situ hybridization correlates with mRNA transcript levels, especially in regions where penetration of probes could pose problems, we performed RT-PCR in two distinct regions of the embryo. We chose the head as a region where all the nups showed strong expression, and the ventral endomesoderm as a region where expression was very low or not detected for some nups. Additionally these two regions were easy to dissect from each other in a practical way given the small size of the embryos (Fig. 8A). We chose 2 nucleoporins from each subcomplex: one with strong expression in the ventral mesoderm and another

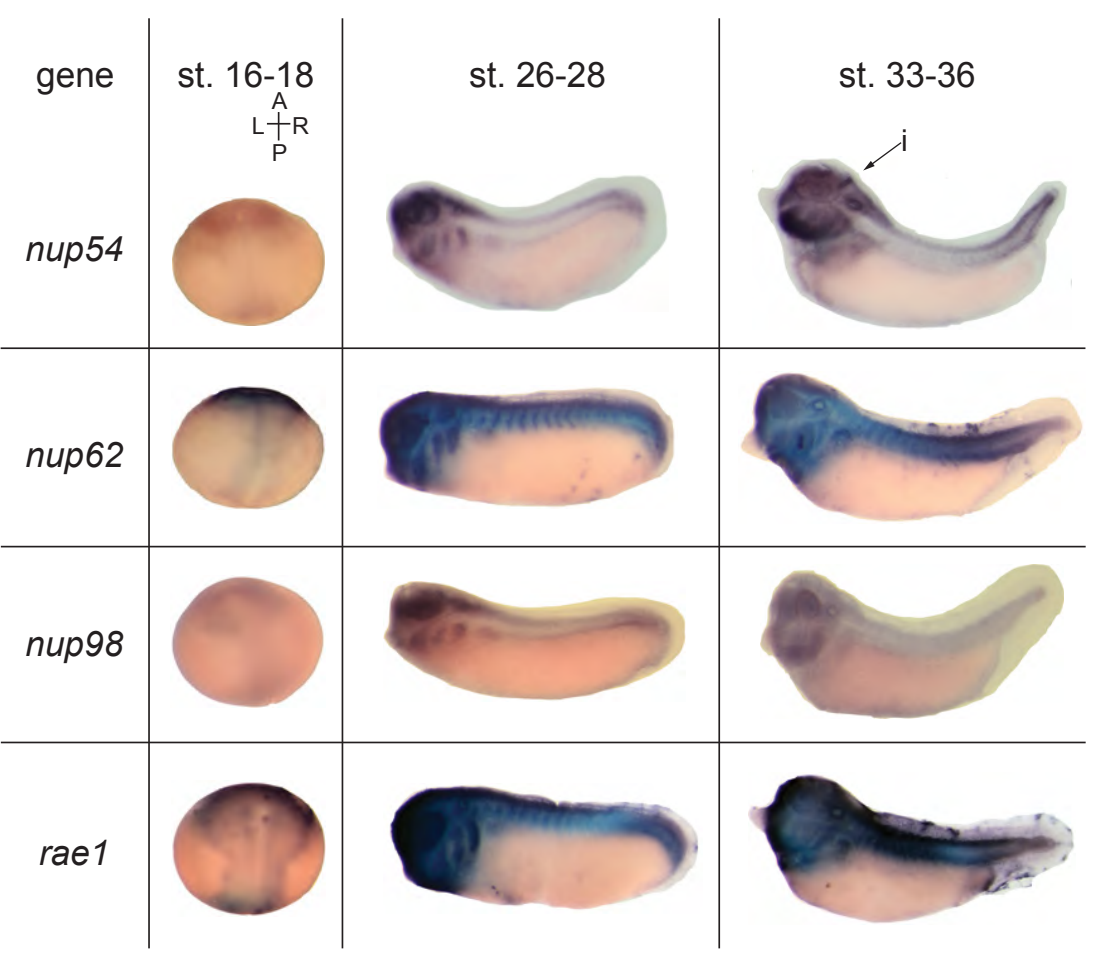

Fig. 5. Expression of Channel nups in developing $\boldsymbol{X}$. tropicalis embryos. Expression was analyzed by whole mount in situ hybridization at 3 different stages of $\mathrm{X}$. tropicalis development (16-18, 26-28 and 33-36, columns). All nups were detected in neural plate region at stages 16-18; brain, eyes and visceral arches at stages 26-28. $i$, isthmus or mid to hindbrain boundary. A: anterior, P: posterior, L: left, R: right. where we could not detect expression by in situ hybridization. For example, for the outer ring nups, seh1 shows strong expression in the ventral mesoderm, whereas for nup133 we could not detect expression in the same region. RT-PCR showed strong expression of both nups in the head region, but we detected expression of seh1 in ventral mesoderm, where nup133 is reduced (Fig. 8B). Although RT-PCR is not quantitative, we were able to qualitatively correlate the expression with the in situ hybridization results, showing nups expression is highly regulated during development.

\section{Discussion}

In general, there are certain regions where nups are expressed across all subcomplexes; these include: the neural folds of early stage embryos, the eyes, visceral (branchial) arches and brain of later stage embryos. Other common areas of expression are the dorso-lateral plate mesoderm and otic vesicle. The question arises as to why signal was consistently and strongly seen in the eyes, brain and visceral arches across all nups. It may be possible that these regions of the embryo have a higher rate of cell proliferation, and therefore a higher rate of nup expression. However, the most striking feature of these expression patterns is the fact that we did not detect ubiquitous expression, despite the fact that nucleoporins are required in every cell. Previous in situ hybridization studies have shown a criteria to classified genes as ubiquitously expressed (Pollet et al., 2005), which is clearly different from the in situ expression patterns we obtained for the nups. Within each subcomplex, one would expect a consistent pattern of expression based on the assumption that all genes within the subcomplex localize to the same structure. Therefore, we would expect a similar expression pattern for nups of the same subcomplex. The structural localization relationship should hold, but the intensity of signal detected for each nup may differ if the stoichiometric relationship between each nup protein in the complex is not $1: 1$. However, interestingly there is variation in localization of expression both between subcomplexes and within each subcomplex. This suggests a greater dynamism and mobility of nucleoporins than previously assumed. It also suggests that each nucleoporin may have several independent functions beyond their roles in the NPC. Whether or not the nup pool involved in function outside the NPC can account for the differential pattern of expression remains unclear.

Nucleoporin proteins of the Nup107-160 complex have been shown to play active roles in mitosis and kinetochore formation (Mishra et al., 2010). In addition, nup107depleted cells were defective in NPC formation, as in its absence several other nup proteins failed to assemble into the NPC complex (Boehmer et al., 2003). This specific role for nup 107 may explain its apparently stronger expression in our in situs, since it plays more than a structural role in a single subcomplex. In another study, seh1 was shown to be necessary for correct chromosome alignment in mitosis and for completion of cytokinesis (Platani et al., 2009). This suggests that in addition to the role that the 107-160 subcomplex has as a unit, individual members of the subcomplex also have unique functions. In a study of neuronal development in mice, nup133 null mutants showed significant defects in neural tube formation that resulted in embryonic lethality (Lupu et al., 2008). In our results, nup133 
A

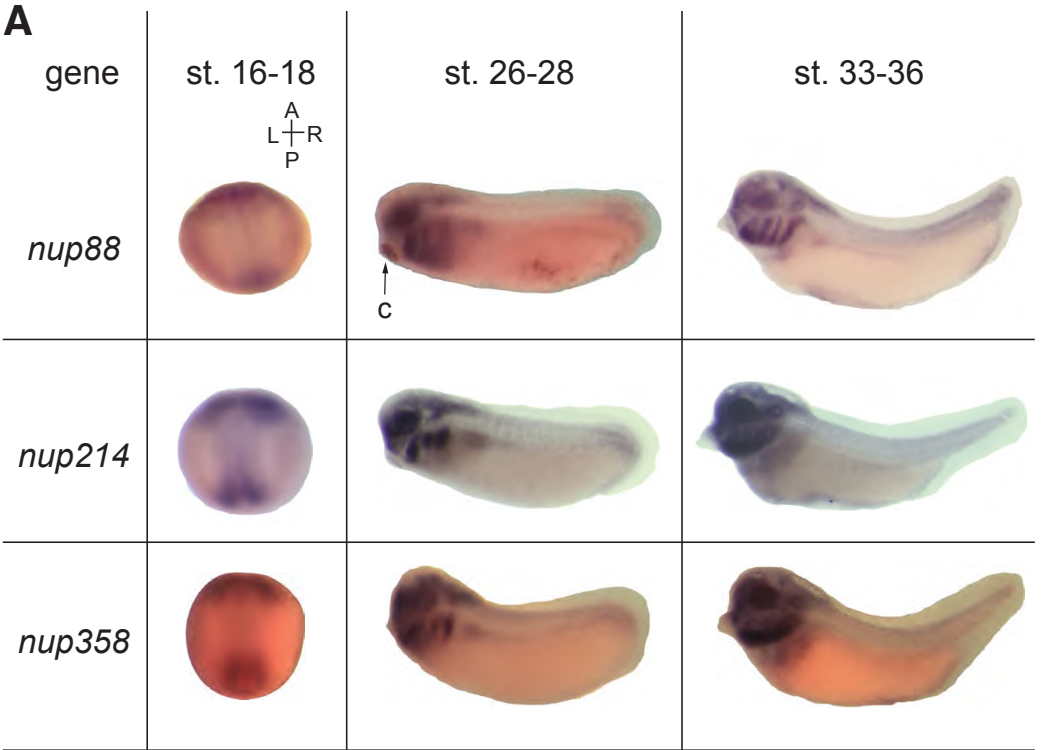

B

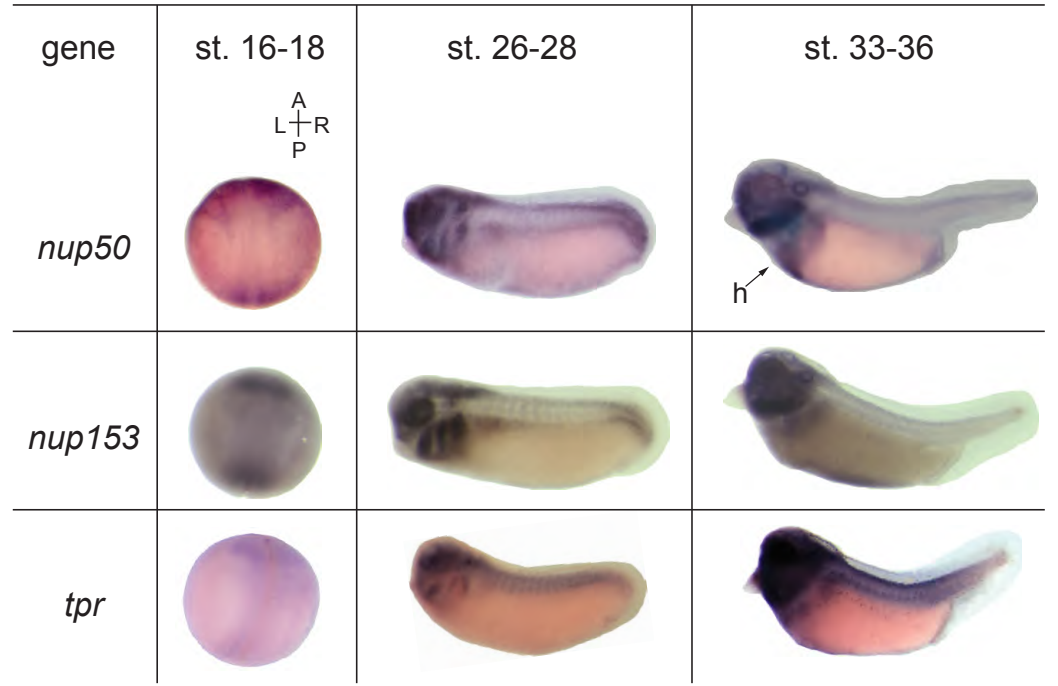

Fig. 6. Expression of cytoplasmic and nuclear basket nups in developing $\boldsymbol{X}$. tropicalis embryos. Expression was analyzed by whole mount in situ hybridization at 3 different stages of $\mathrm{X}$. tropicalis development (16-18, 26-28 and 33-36, columns). (A) Cytoplasmic nups and (B) Nuclear basket nups expression patterns. Neural tube signal was detected all nups at stages 16-18. At stages 26-28 and 33-36 transcript expression was detected in the head, eyes, and visceral arches in all nups. $h$, heart progenitor field; c, cement gland. A: anterior, P: posterior, L: left, R: right.

for protein trafficking to the Golgi apparatus (Niu et al., 2014). These results may point to a similar role for sec13 in Xenopus, given its strong expression in the eye regions in our in situs. These studies and many others showed that nucleoporins play an important role in embryonic development (Raices and D'Angelo, 2012).

Interestingly, functional studies on nucleoporins seemed to have focused largely on the scaffold nucleoporins, leaving a large portion of the nup family genes still open for further experimentation. For instance, $t p r$, a member of the nuclear ring subcomplex, has been shown to play a role in the formation of heterochromatin exclusion zones proximal to NPCs in somatic cells (Krull et al., 2010). In addition, rae1 null mutants in mice were found to be embryonic lethal. Moreover, haploinsufficient rae1 mutants were shown to have mitotic checkpoint and chromosomal segregation abnormalities, similar to the mutant phenotypes found in functional studies of the scaffold subcomplex nups (Babu et al., 2003). Furthermore, nup62 depletion induced cell cycle arrest and affected centriole and centrosome function (Hashizume et al., 2013).

Given the diverse roles of nucleoporins determined thus far, it can be expected that other nups may possess additional functions beyond a structural role in the NPC. Our study suggests that from a developmental perspective, there may be certain embryonic regions that are differentially affected by nucleoporin function. However, further studies are needed to illuminate what these potential roles are.

Overall, our results have shown that nup expression is subject to a high degree of spatial regulation and transcripts showed a unique expression pattern (Fig. 3), with strong expression in the mid to hind brain boundary at stages 33-36, indicating that it may be a candidate for further study of neuronal development in Xenopus as well. Given their similar expression patterns within the brain to nup 133, nups 54, 88, 93 and 155 may also be candidates for further study in this regard. Recently, Niu et al., showed that sec13 plays independent functional roles in the NPC (affecting retinal development) and in the COPII complex of the ER, which is responsible

Fig. 7. Expression of Transmembrane nups in developing $\boldsymbol{X}$. tropicalis embryos. Expression was analyzed by whole mount in situ hybridization at 3 different stages of $\mathrm{X}$. tropicalis development (16-18, 26-28 and 33-36, columns). At stages 16-18 expression was detected for all genes in the neural tube. All nups showed expression in the head, eyes, and visceral arches at stages 26-28 and 33-36. A: anterior, P: posterior, L: left, R: right.

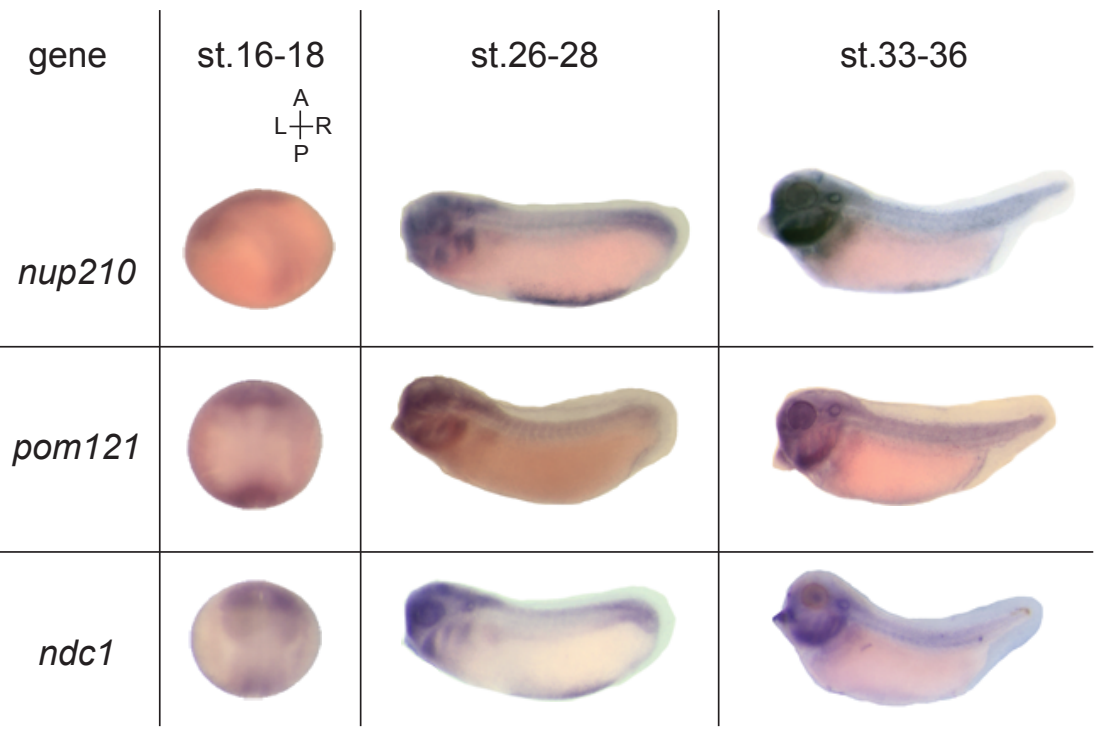


are produced in a much higher amount in specific regions of the embryo, even if the ubiquitous presence of nucleoporins is assumed in cells. Furthermore, absolute quantification of nup transcripts per embryo also shows dynamic transcriptional regulation during early development (Fig. 2, (Owens et al., 2016). Knowledge of these expression patterns provides great insight into potential directions of experimentation and study of nucleoporin localization and function.

\section{Materials and Methods}

\section{Animal husbandry}

$X$. tropicalis were housed and cared for in our aquatics facility according to established protocols that were approved by Yale IACUC.

\section{RNA-seq data analysis}

RNA-seq profiles of nucleoporins during $X$. tropicalis development were obtained from http://genomics.crick.ac.uk/cgi-bin/profile-search.exe, (Owens et al., 2016). Graphs in Fig. 2 show the expression profiles grouped by subcomplex. This RNA-Seq profile represent an absolute quantification of transcripts per embryo from 0 to 66 hours post fertilization (horizontal axis) with $95 \%$ confidence interval depicted in the shaded region.

\section{Obtaining and fixing embryos}

WT Embryos were obtained from Xenopus natural matings (del Viso and Khokha, 2012). Embryos were allowed to grow in 1/9X MR supplemented with $50 \mathrm{ug} / \mathrm{ml}$ of gentamycin to the desired stages (16-18, 26-28 and 33$36)$, that were scored using the Normal Table of Xenopus laevis (Daudin) (Nieuwkoop and Faber, 1994). Embryos were fixed in MEMFA (0.1M MOPS (pH7.4), 2mM EGTA, 1mM MgSO4, 3.7\% v/v formaldehyde) for 2 hours at room temperature, and subsequently dehydrated in $100 \%$ ethanol overnight. Vitelline membrane was removed manually using forceps. Stocks were stored at $-20^{\circ} \mathrm{C}$. Around 1000 embryos of each range of stages were fixed and used for in situ hybridizations.

\section{Whole-mount in situ hybridization}

We obtained clones from our own cDNA library stocks (Sanger Clone Library), Open Biosystems (Thermo Scientific) and the Harland Lab at the University of California-Berkeley (Table 1). A portion of the nup188 clone was amplified by PCR using the primers Nup188F1: CCAGCAGGATCTTGTAGACACAGC and Nup188R1: CAGGGGATAAGTTGAGGATTGCAT to obtain a $1 \mathrm{~kb}$ segment for in situ probe synthesis. RNA probe synthesis was done via in vitro transcription using T7 High Yield RNA Synthesis Kit (E2040S) from New England Biolabs, using digoxigenin labeled dUTP. To synthesize RNA sense probes we used the same clones and the Maxiscript SP6 (AM1308) from Ambion, using digoxigenin labeled dUTP. The wholemount in situ hybridization procedure was done according to the standard protocol (Khokha et al., 2002). Embryos were stained with BM Purple at room temperature or $4^{\circ} \mathrm{C}$ depending on ideal conditions for each probe. Embryos were then bleached and suspended in glycerol for long-term storage. Images were taken with a Canon DS126201 camera and Zeiss Discovery.V8 SteREO scope. Exposure settings were kept constant for all images. In situ hybridizations were done between 2 and 3 times for each nucleoporin. After we detected strong signal for each gene, we fixed half of the embryos and continued developing the other half to asses if the signal would become ubiquitous. Developing embryos further did not change the original pattern of expression and in some cases lead to background due to inappropriate precipitation of the substrate. As negative controls we used sense RNA probes for at least one representative of each subcomplex (Supplementary Fig. S1). Sense probes were developed for the same amount of time as the respective antisense probe.

\section{RT-PCR assays}

Embryos were raised to the desired stages (26-28 or 33-36) and two regions were isolated: the head and the ventral mesoderm (Fig. 8A). Tissue was immediately immersed in Trizol Reagent (Life Technologies) and RNA was extracted following the manufacturer's instructions. The Superscript ${ }^{\mathrm{TM}} \mathrm{IV}$ Reverse Transcriptase (Thermofisher) was used to synthesize cDNA using $1 \mu \mathrm{g}$ of total RNA from each tissue. PCR was performed using Phusion High

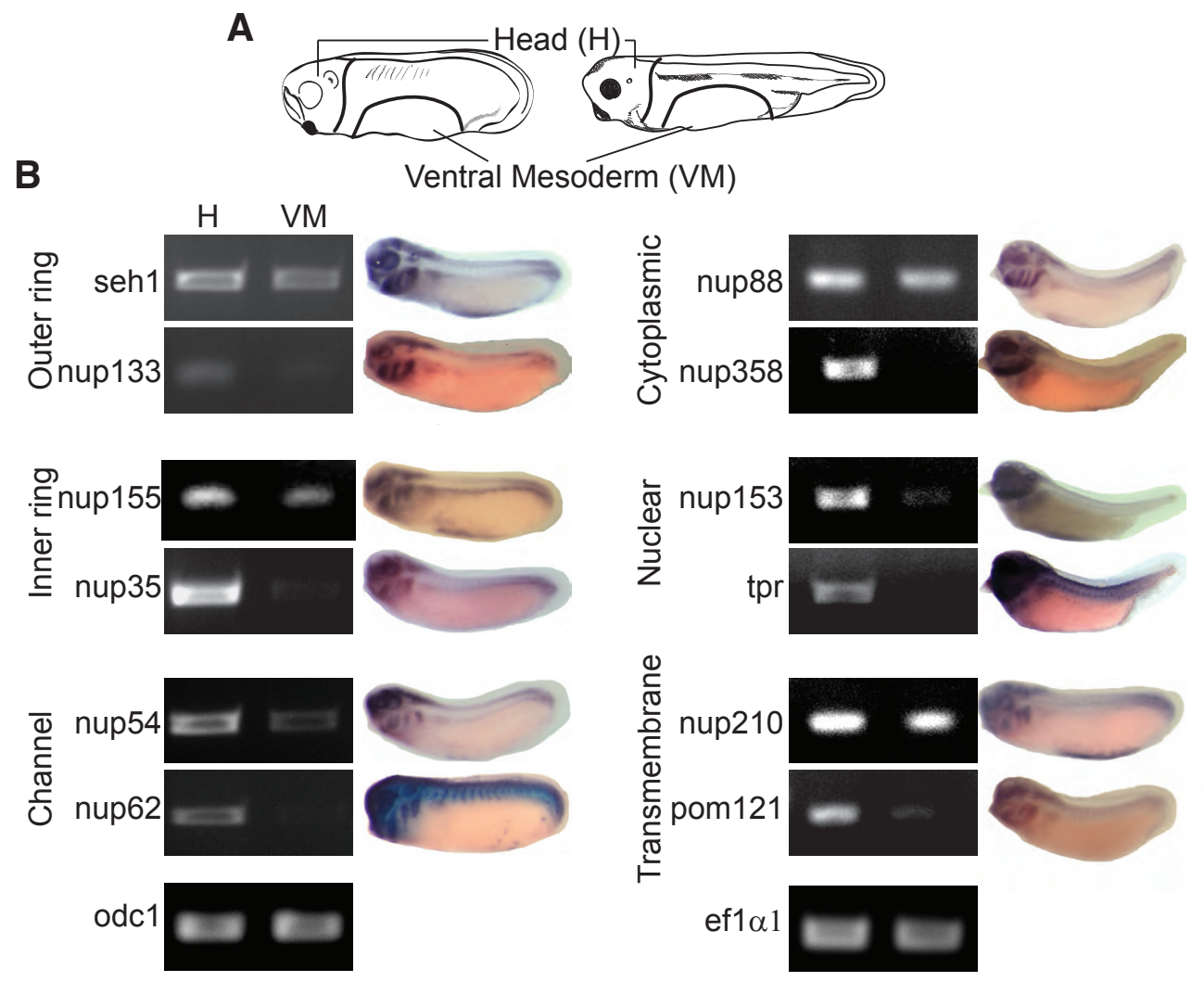

Fig. 8. Correlation of nup expression between in situ hybridization and RTPCR. (A) Schematics of stages 28 and 33 depicting Head $(H)$ and ventral mesoderm (VM) regions that were dissected and used for RNA extractions and subsequent RT-PCR. (B) Expression by RT-PCR of 2 nups for each subcomplex, in each of the dissected tissues. In situ hybridization images from previous figures are shown for illustration. Odc1 (ornithine decarboxylase 1) and ef $1 \alpha 1$ (eukaryotic translation elongation factor 1 alpha 1) were used as positive controls of RT-PCR. The primer sequences for each nup are shown in Table 1. 
TABLE 1

\section{LIST OF ALL NUP GENES TESTED WITH CLONE ID INFORMATION AND PRIMERS USED FOR RT-PCR}

\begin{tabular}{|c|c|c|}
\hline Gene Name & Clone ID & RT-PCR primers \\
\hline Nup35 & TEgg013j23 & $\begin{array}{l}\text { XtNup35F1: 5' CATGGAGCCTATGGGTGCTG } \\
\text { XtNup35R1: 5' CAACAGACTGTGTAGGAGTCCCTA }\end{array}$ \\
\hline Nup37 & TGas082o16 & \\
\hline Nup43 & TEgg125d07 & \\
\hline Nup50 & TNeu054d12 & \\
\hline Nup54 & TGas114o17 & $\begin{array}{l}\text { XtNup54F1: 5' AACCAATCTGTTCAAAGCACGCC } \\
\text { XtNup54R1: 5' TGTTGCTTTGTCATCTGATCTTGGA }\end{array}$ \\
\hline Nup62 & 7605025 & $\begin{array}{l}\text { XtNup62F1: 5' AGGCGGTCTTACCTTTGGTG } \\
\text { XtNup62R1: 5' GCGTTGACCTGAGTTGCTTG }\end{array}$ \\
\hline Nup85 & TNeu102j12 & \\
\hline Nup88 & TGas106b12 & $\begin{array}{l}\text { XtNup88F1: 5' CTGGAGATTCCTAAACGCTGGGG } \\
\text { XtNup88R1: 5' GCAATGGTATCGGGATGGGC }\end{array}$ \\
\hline Nup93 & 5379233 & \\
\hline Nup96 & 8889890 & \\
\hline Nup98 & 7686621 & \\
\hline Nup107 & 8850047 & \\
\hline Nup133 & 7623820 & $\begin{array}{l}\text { XtNup133F2: 5' AAAGATTAATCAACGTATTCTGCCG } \\
\text { XtNup133R2: 5' TATCTCCTTGTGCTTCAAATGGAAT }\end{array}$ \\
\hline Nup153 & 8851980 & $\begin{array}{l}\text { XtNup153F1: 5' CAAACGCTCAGCAGTATGGGG } \\
\text { XtNup153R1: 5' TCCTAAGGTGACCTTTACAGGGG }\end{array}$ \\
\hline Nup155 & TGas122g21 & $\begin{array}{l}\text { XtNup155F1: 5' GTGCTTGCTGTGCTCAGTTTGC } \\
\text { XtNup155R1: 5' GGCAAGGAATATAGCGGGTCTG }\end{array}$ \\
\hline Nup160 & 8851700 & \\
\hline Nup188 & 7675066 & \\
\hline Nup205 & 7684539 & \\
\hline Nup210 & 7554222 & $\begin{array}{l}\text { XtNup210F1: 5' TTAGAGCTAAAATGCTGGAGCCG } \\
\text { XtNup210R1: 5' TGGGAACGTAAGAATTTTAGGGG }\end{array}$ \\
\hline Nup214 & 8851888 & \\
\hline Nup358 & 7586397 & $\begin{array}{l}\text { XtNup358F1: 5' ACAGATTTAGAATGGTGCTCCCG } \\
\text { XtNup358R1: 5' AAGTCTTGAAGTTTCCTGGGGC }\end{array}$ \\
\hline Ndc1 & TEgg045I09 & \\
\hline Rae1 & 7604803 & \\
\hline Sec13 & TGas091g04 & \\
\hline Seh1 & TGas096I04 & $\begin{array}{l}\text { XtSeh1F1: 5' GGGACAAAAGTGAAAATGGCG } \\
\text { XtSeh1R1: 5' TGTGCCAGTGATGTTCCAACTTAC }\end{array}$ \\
\hline Tpr & TNeu065h23 & $\begin{array}{l}\text { XtTprF1: 5' AATGGAATCCGTGAAAGCCCG } \\
\text { XtTprR1: 5' TTGTGAAGCTCCTCCACTGCCC }\end{array}$ \\
\hline Pom121 & TGas001m22 & $\begin{array}{l}\text { XtPom121F2: 5' ATCTGGAACCAGCAGCCTTC } \\
\text { XtPom121R2: 5' ACCAAACGTAGGCTGAGTGG }\end{array}$ \\
\hline ODC1 & & $\begin{array}{l}\text { ODC1-F: 5' TGTTCTGCGCATAGCAACTG } \\
\text { ODC1-R: 5' ACATCGTGCATCTGAGACAGC }\end{array}$ \\
\hline Ef1a1 & & 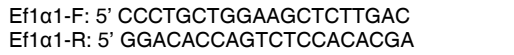 \\
\hline
\end{tabular}

Fidelity DNA polymerase (New England Biolabs) and the following program: $98^{\circ} \mathrm{C}$ for $30 \mathrm{~s}, 22$ cycles of $98^{\circ} \mathrm{C}$ for $15 \mathrm{~s}, 60-66^{\circ} \mathrm{C}$ for $30 \mathrm{~s}, 72^{\circ} \mathrm{C}$ for $45 \mathrm{sec}$, and a final elongation at $72^{\circ} \mathrm{C}$ for $5 \mathrm{~min}$. Annealing temperature varied according to each pair of primers. Odc1 (ornithine decarboxylase 1) and ef1 $\alpha$ (eukaryotic translation elongation factor 1 alpha 1) were used as positive controls of RT-PCR. The primers used for each nup are shown in Table 1.

\section{Acknowledgements}

We thank Sarah Kubek and Michael Slocum for frog husbandry and John Griffin and Maura Lane for help in taking and processing images. We also thanks Richard Harland and Edivinia Pangilinan for kindly providing some of the nups clones. This work was supported by NIH/NHLBI R01HL124402 to Mustafa Khokha. Mustafa Khokha is a Mallinckrodt Scholar.

\section{References}

BABU, J.R., JEGANATHAN, K.B., BAKER, D.J., WU, X., KANG-DECKER, N. and VAN DEURSEN, J.M. (2003). Rae1 is an essential mitotic checkpoint regulator that cooperates with Bub3 to prevent chromosome missegregation. J Cell Biol 160: 341-353.
BOEHMER, T., ENNINGA, J., DALES, S., BLOBEL, G. and ZHONG, H. (2003) Depletion of a single nucleoporin, Nup107, prevents the assembly of a subset of nucleoporins into the nuclear pore complex. Proc Natl Acad Sci USA 100: 981-985.

DEL VISO, F. and KHOKHA, M. (2012). Generating diploid embryos from Xenopus tropicalis. Methods Mol Biol 917: 33-41.

DINUNZIO, F., FRICKE, T., MICCIO, A., VALLE-CASUSO, J.C., PEREZ, P., SOUQUE, P., RIZZI, E., SEVERGNINI, M., MAVILIO, F., CHARNEAU, P. et al., (2013). Nup153 and Nup98 bind the HIV-1 core and contribute to the early steps of HIV-1 replication. Virology 440: 8-18.

FAKHRO, K.A., CHOI, M., WARE, S.M., BELMONT, J.W., TOWBIN, J.A., LIFTON, R.P., KHOKHA, M.K. and BRUECKNER, M. (2011). Rare copy number variations in congenital heart disease patients identify unique genes in left-right patterning. Proc Natl Acad Sci USA 108: 2915-2920.

FONTOURA, B.M., BLOBEL, G. and MATUNIS, M.J. (1999). A conserved biogenesis pathway for nucleoporins: proteolytic processing of a 186-kilodalton precursor generates Nup98 and the novel nucleoporin, Nup96. J Cell Biol 144: 1097-1112.

GROSSMAN, E., MEDALIA, O. and ZWERGER, M. (2012). Functional architecture of the nuclear pore complex. Annu Rev Biophys 41: 557-584.

HASHIZUME, C., MOYORI, A., KOBAYASHI, A., YAMAKOSHI, N., ENDO, A. and WONG, R.W. (2013). Nucleoporin Nup62 maintains centrosome homeostasis. Cell Cycle 12: 3804-3816.

KHOKHA, M.K., CHUNG, C., BUSTAMANTE, E.L., GAW, L.W., TROTT, K.A., YEH, J., LIM, N., LIN, J.C., TAVERNER, N., AMAYA, E. et al., (2002). Techniques and probes for the study of Xenopus tropicalis development. Dev Dyn 225: 499-510.

KRULL, S., DORRIES, J., BOYSEN, B., REIDENBACH, S., MAGNIUS, L., NORDER, H., THYBERG, J. and CORDES, V.C. (2010). Protein Tpr is required for establishing nuclear pore-associated zones of heterochromatin exclusion. EMBOJ29:1659-1673.

LIN, D.H., STUWE, T., SCHILBACH, S., RUNDLET, E.J., PERRICHES, T., MOBBS, G., FAN, Y., THIERBACH, K., HUBER, F.M., COLLINS, L.N. et al., (2016). Architecture of the symmetric core of the nuclear pore. Science 352: aaf1015.

LUPU, F., ALVES, A., ANDERSON, K., DOYE, V. and LACY, E. (2008). Nuclear pore composition regulates neural stem/progenitor cell differentiation in the mouse embryo. Dev Cell 14: 831-842.

LUSK, C.P., BLOBEL, G. and KING, M.C. (2007). Highway to the inner nuclear membrane: rules for the road. Nat Rev Mol Cell Biol 8: 414-420.

MISHRA, R.K., CHAKRABORTY, P., ARNAOUTOV, A., FONTOURA, B.M. andDASSO, M. (2010). The Nup107-160 complex and gamma-TuRC regulate microtubule polymerization at kinetochores. Nat Cell Biol 12: 164-169.

NIEUWKOOP, P.D. and FABER, J. (1994). Normal table of Xenopus laevis (Daudin): a systematical and chronological survey of the development from the fertilized egg till the end of metamorphosis. Garland Publishing, Inc.

NIU, X., HONG, J., ZHENG, X., MELVILLE, D.B., KNAPIK, E.W., MENG, A. and PENG, J. (2014). The nuclear pore complex function of Sec13 protein is required for cell survival during retinal development. J Biol Chem 289: 11971-11985.

ONISCHENKO, E. and WEIS, K. (2011). Nuclear pore complex-a coat specifically tailored for the nuclear envelope. Curr Opin Cell Biol 23: 293-301.

OWENS, N.D., BLITZ, I.L., LANE, M.A., PATRUSHEV, I., OVERTON, J.D., GILCHRIST, M.J., CHO, K.W. and KHOKHA, M.K. (2016). Measuring Absolute RNA Copy Numbers at High Temporal Resolution Reveals Transcriptome Kinetics in Development. Cell Rep 14: 632-647.

PLATANI, M., SANTARELLA-MELLWIG, R., POSCH, M., WALCZAK, R., SWEDLOW, J.R. and MATTAJ, I.W. (2009). The Nup107-160 nucleoporin complex promotes mitotic events via control of the localization state of the chromosome passenger complex. Mol Biol Cell 20: 5260-5275.

POLLET, N., MUNCKE, N., VERBEEK, B., LI, Y., FENGER, U., DELIUS, H. and NIEHRS, C. (2005). An atlas of differential gene expression during early Xenopus embryogenesis. Mech Dev 122: 365-439.

RAICES, M. and D'ANGELO, M.A. (2012). Nuclear pore complex composition: a new regulator of tissue-specific and developmental functions. Nat Rev Mol Cell Biol 13: 687-699.

THEERTHAGIRI, G., EISENHARDT, N., SCHWARZ, H. and ANTONIN, W. (2010). The nucleoporin Nup188 controls passage of membrane proteins across the nuclear pore complex. J Cell Biol 189: 1129-1142. 


\section{Further Related Reading, published previously in the Int. J. Dev. Biol.}

Developmental expression of the N-myc downstream regulated gene (Ndrg) family during Xenopus tropicalis embryogenesis Chao Zhong, Yan-Kuan Zhou, Shan-Shan Yang, Jun-Fang Zhao, Xiao-Long Zhu, Zhen-Huang Chen, Pei-Chao Chen, Li-Quan Huang and Xiao Huang

Int. J. Dev. Biol. (2015) 59: 511-517

http://dx.doi.org/10.1387/ijdb.150178xh

The importance of cartilage to amphibian development and evolution

Christopher S. Rose

Int. J. Dev. Biol. (2014) 58: 917-927

http://dx.doi.org/10.1387/ijdb.150053cr

Comparative expression analysis of cysteine-rich intestinal protein family members crip1, 2 and 3 during Xenopus laevis embryogenesis

Annemarie Hempel and Susanne J. Kühl

Int. J. Dev. Biol. (2014) 58: 841-849

http://dx.doi.org/10.1387/ijdb.140270sk

Two different vestigial like 4 genes are differentially expressed during Xenopus laevis development

María-Guadalupe Barrionuevo, Manuel J. Aybar and Celeste Tríbulo

Int. J. Dev. Biol. (2014) 58: 369-377

http://dx.doi.org/10.1387/ijdb.130353ct

The lysophosphatidic acid (LPA) and sphingosine-1-phosphate (S1P) receptor gene families: cloning and comparative expression analysis in Xenopus laevis Karine Massé, Jun-ichi Kyuno, Surinder Bhamra and Elizabeth A. Jones

Int. J. Dev. Biol. (2010) 54: 1361-1374

http://dx.doi.org/10.1387/ijdb.103068km

Expression of Bmp ligands and receptors in the developing Xenopus retina Jennifer C. Hocking and Sarah McFarlane

Int. J. Dev. Biol. (2007) 51: 161-165

http://dx.doi.org/10.1387/ijdb.062185jh

5 yr ISI Impact Factor $(2013)=2.879$

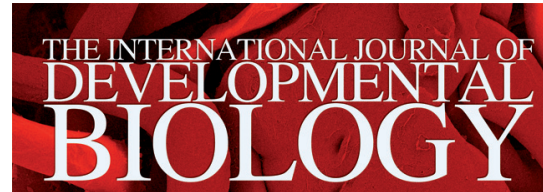

Volume 57 Nos. 2/3/4

Special Issue
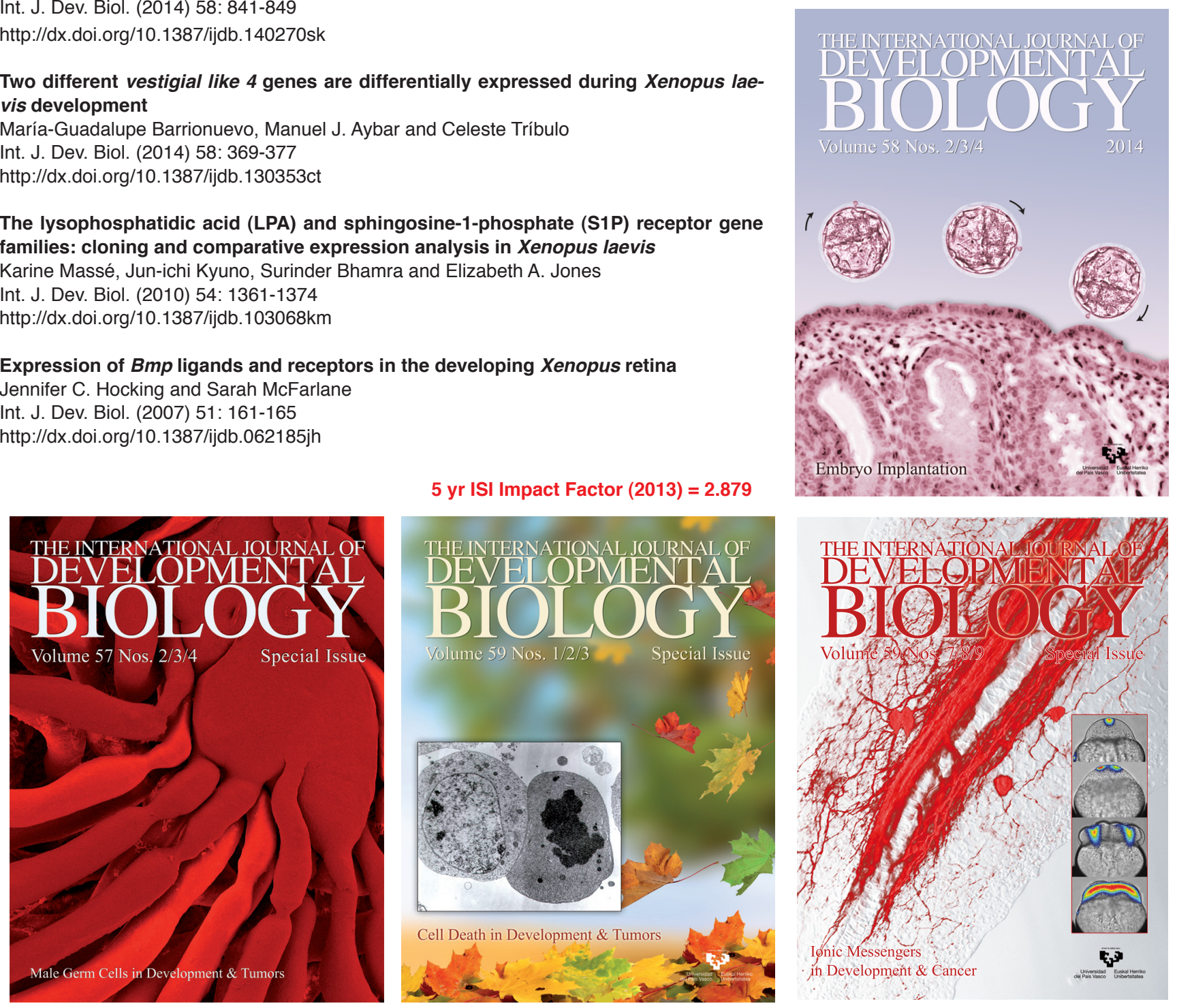